\title{
The initial boundary value problem for the nonlinear parabolic equation in unbounded domain
}

\author{
Lech Zarȩba
}

\begin{abstract}
In this paper we consider the mixed problem for the equation $u_{t t}+$ $A_{1} u+A_{2}\left(u_{t}\right)+g\left(u_{t}\right)=f(x, t)$ in unbounded domain, where $A_{1}$ is a linear elliptic operator of the fourth order and $A_{2}$ is a nonlinear elliptic operator of the second order. Under natural assumptions on the equation coefficients and $f$ we proof existence of a solution. This result contains, as a special case, some of known before theorems of existence. Essentially, in difference up to previous results we prove theorems of existence without the additional assumption on behavior of solution at infinity.
\end{abstract}

Mathematics Subject Classification (2000). Primary 35K55, Secondary 35K60.

Keywords. Initial boundary value problem, Nonlinear parabolic equation.

\section{Introduction}

Initial boundary value problems for nonlinear parabolic equations in unbounded domain were studied by many authors. Most of the known results about the existence and uniqueness require additional assumptions on the behavior at infinity by space variables $x$. So, such type results for boundary value problems of nonlinear parabolic equations of the fourth order with the second order time derivative in $[18,27]$ are received. In papers $[8,9,17,21,24]$ similar results for the Cauchy problem of parabolic nonlinear equations was obtained. In [25] the authors have shown the uniqueness of a weak solution in the class of functions which do not grow faster than the function $e^{a|x|^{\alpha}}$ for $|x| \rightarrow \infty$.

The main goal of the given article is to present such conditions for initial boundary value problems of nonlinear parabolic equations with nonlinearities in unbounded domains by space variables, at which the existence of solution does not depend on its behaviour at $|x| \rightarrow+\infty$.

We consider an initial boundary value problem in the form which will allow to generalize above mentioned results about existence of solutions. Namely, we 
consider the equation

$$
\begin{aligned}
A(u) \equiv & u_{t t}(x, t)+\sum_{i, j, k, l=1}^{n}\left(a_{i j}^{k l}(x) u_{x_{i} x_{j}}(x, t)\right)_{x_{k} x_{l}} \\
& -\sum_{i, j=1}^{n}\left(a_{i j}(x) u_{x_{i}}(x, t)\right)_{x_{j}}-\sum_{i=1}^{n}\left(b_{i}(x)\left|u_{t x_{i}}(x, t)\right|^{p-2} u_{t x_{i}}(x, t)\right)_{x_{i}} \\
& +a_{0}(x) u(x, t)+c(x)\left|u_{t}(x, t)\right|^{q-2} u_{t}(x, t)=f(x, t)
\end{aligned}
$$

with the following boundary and initial conditions

$$
\begin{aligned}
& \left.u\right|_{S_{T}}=0,\left.\quad \frac{\partial u}{\partial \nu}\right|_{S_{T}}=0, \\
& \left.u\right|_{t=0}=u_{0}(x),\left.\quad u_{t}\right|_{t=0}=u_{1}(x),
\end{aligned}
$$

where $S_{T}=\partial \Omega \times(0, T)$ and $\nu$ is an outward normal vector for $S_{T}, p>2, q>2$.

We assume that the coefficients of (1.1) satisfies the following conditions:

$\left(\mathbf{A}_{1}\right) \quad a_{i j}^{k l} \in B^{2}(\Omega) ; \quad a_{i j}^{k l}(x) \stackrel{\text { a.e. }}{=} a_{k l}^{i j}(x),(x \in \Omega), \quad i, j, k, l=1, \ldots, n$, $\sum_{i, j, k, l=1}^{n} a_{i j}^{k l}(x) \xi_{i j} \xi_{k l} \stackrel{\text { a.e. }}{\geq} a_{2} \sum_{i, j=1}^{n} \xi_{i j}^{2},(x \in \Omega), \quad \xi \in R^{n(n+1) / 2}$ with a constant $a_{2}>0$; $\left(\mathbf{A}_{\mathbf{2}}\right) \quad a_{i j} \in B^{1}(\Omega), i, j=1, \ldots, n, \quad a_{i j}(x) \stackrel{\text { a.e. }}{=} a_{j i}(x),(x \in \Omega)$, $\sum_{i j=1}^{n} a_{i j}(x) \xi_{i} \xi_{j} \stackrel{\text { a.e. }}{\geq} a_{1} \sum_{i=1}^{n} \xi_{i}^{2},(x \in \Omega), \quad \xi \in R^{n}$ with a constant $a_{1}>0 ;$

$\left(\mathbf{A}_{\mathbf{3}}\right) \quad a_{0} \in L^{\infty}(\Omega), \quad a_{0}(x) \stackrel{\text { a.e. }}{\geq} a_{0} \geq 0,(x \in \Omega)$ with a constant $a_{0}$;

(B) $\quad b_{i} \in B^{1}(\Omega), \quad b_{i}(x) \stackrel{\text { a.e. }}{\geq} b_{0}>0,(x \in \Omega)$ with a constant $b_{0}$;

(C) $c \in L^{\infty}(\Omega), \quad c(x) \stackrel{\text { a.e. }}{\geq} c_{0}>0,(x \in \Omega)$ with a constant $c_{0}$.

Denote

$$
\begin{aligned}
H_{0,1}^{2}\left(\Omega^{R}\right) & =\left\{u: u \in H^{2}\left(\Omega^{R}\right) ;\left.u\right|_{\partial \Omega \cap B_{R}}=0,\left.\frac{\partial u}{\partial \nu}\right|_{\partial \Omega \cap B_{R}}=0\right\}, \\
W_{0,1}^{1, p}\left(\Omega^{R}\right) & =\left\{u: u \in W^{1, p}\left(\Omega^{R}\right),\left.u\right|_{\partial \Omega \cap B_{R}}=0\right\}, \\
H_{0, \text { loc }}^{k}(\bar{\Omega}) & =\left\{u \in H_{0,1}^{k}\left(\Omega^{R}\right), \forall_{R>1}\right\}, \quad(k=1,2), \\
L_{\text {loc }}^{2}(\bar{\Omega}) & =\left\{u \in L^{2}\left(\Omega^{R}\right), \forall_{R>1}\right\}, \quad W_{0, \text { loc }}^{1, p}(\bar{\Omega})=\left\{u: u \in W_{0,1}^{1, p}\left(\Omega^{R}\right), \forall_{R>1}\right\}, \\
B^{1}(\Omega) & =\left\{u: u, u_{x_{i}} \in L^{\infty}(\Omega), \quad i=1,2, \ldots, n\right\}, \\
B^{2}(\Omega) & =\left\{u: u, u_{x_{i}}, u_{x_{i} x_{j}} \in L^{\infty}(\Omega), \quad i, j=1,2, \ldots, n\right\} .
\end{aligned}
$$

In work is proved that if the above conditions are executed that there exist solutions without additional conditions at infinity by space variables.

Notice also that, similar problems is actual for another types equations, for example for the nonlinear parabolic equations with the first derivative with 
respect to time considered in unbounded domain. So, in [5] it was showed that the uniqueness of Cauchy problem solution for the equation

$$
u_{t}-\triangle^{m}\left(|u|^{p-2} u\right)+c|u|^{q-2} u=0
$$

with $m=1, c=1, p=2, q>2$ does not depend on its behaviour for $|x| \rightarrow+\infty$. Similar results were obtained for $m \geq 1, p=2, c>0, q>2$ in $[2-4,12,13,15,16$, $22,23,26]$. In [11] it was showed that the equation

$$
u_{t}-\operatorname{div}\left(|D u|^{p-2} D u\right)=0
$$

also possesses analogical property. In [10] it was showed that if $m=1, c \geq 0$, $p>2$ in (1.5), then the solution of Cauchy problem exists and it is unique in the class of functions which do not grow faster than $|x|^{s}$, as $|x| \rightarrow+\infty$, where $s$ depends on $p$ and $n$. A similar result was obtained in $[1,6,7,12,19]$ for the degenerate quasi-linear parabolic equations of types (1.4), (1.5). We can observe, that our results is a partial analog of these researches for the case of equations with the second derivative by time.

\section{The main results}

Let $\Omega \subset \mathbb{R}^{n}$ be an unbounded domain and $\partial \Omega \in C^{1}, \Omega \cap K_{R}=\Omega^{R}$ be a regular domain for all $R>1$, where for all $R>1, K_{R}=\left\{x \in \mathbb{R}^{n},|x|<R\right\}$ and $Q_{T}=$ $\Omega \times(0, T), T<+\infty$. In the domain $Q_{T}$ we shall consider the problem (1.1)-(1.3).

In the sequel, we take the assumptions $\left(\mathbf{A}_{\mathbf{1}}\right),\left(\mathbf{A}_{\mathbf{2}}\right),\left(\mathbf{A}_{\mathbf{3}}\right),(\mathbf{B}),(\mathbf{C})$.

Under these assumptions we will obtain the existence of weak solution of problem (1.1)-(1.3).

Definition 2.1. We call a function $u$ weak solution of the problem (1.1)-(1.3) if

$$
\begin{aligned}
& u \in L^{2}\left((0, T) ; H_{0, \mathrm{loc}}^{2}(\bar{\Omega})\right), \\
& u_{t} \in L^{p}\left((0, T) ; W_{0, \mathrm{loc}}^{1, p}(\bar{\Omega})\right) \cap L^{q}\left((0, T) ; L_{\mathrm{loc}}^{q}(\bar{\Omega})\right) \cap C\left([0, T] ; L_{\mathrm{loc}}^{2}(\bar{\Omega})\right),
\end{aligned}
$$

and $u$ satisfies the following integral equation

$$
\begin{aligned}
& \int_{\Omega} u_{t}(x, \tau) v(x, \tau) d x+\int_{Q_{\tau}}\left[-u_{t} v_{t}+\sum_{i, j, k, l=1}^{n} a_{i j}^{k l}(x) u_{x_{i} x_{j}} v_{x_{k} x_{l}}\right. \\
& \quad+\sum_{i, j=1}^{n} a_{i j}(x) u_{x_{i}} v_{x_{j}}+a_{0}(x) u v+\sum_{i=1}^{n} b_{i}(x)\left|u_{x_{i} t}\right|^{p-2} u_{x_{i} t} v_{x_{i}} \\
& \left.\quad+c(x)\left|u_{t}\right|^{q-2} u_{t} v\right] d x d t=\int_{Q_{\tau}} f(x, t) v d x d t+\int_{\Omega} u_{1}(x) v(x, 0) d x \\
& \forall v \in L^{2}\left((0, T) ; H_{0, \mathrm{loc}}^{2}(\bar{\Omega})\right) \cap L^{q}\left((0, T) ; L_{\mathrm{loc}}^{q}(\bar{\Omega})\right) \cap L^{p}\left((0, T) ; W_{0, \mathrm{loc}}^{1, p}(\bar{\Omega})\right), \\
& v_{t} \in L^{2}\left((0, T) ; L_{\mathrm{loc}}^{2}(\bar{\Omega})\right), \forall \tau \in(0, T],
\end{aligned}
$$


where supp $v$ is bounded. Moreover, $u$ satisfies the condition $\left.u\right|_{t=0}=u_{0}(x)$.

Let $V_{\text {loc }}(\bar{\Omega})=\left\{v \in V\left(\Omega^{R}\right), \forall_{R>1}\right\}$, where

$$
\begin{aligned}
V\left(\Omega^{R}\right)= & \left\{v: v \in H_{0,1}^{2}\left(\Omega^{R}\right) \cap L^{2(q-1)}\left(\Omega^{R}\right),\right. \\
& \left.v_{x_{i}} \in L^{2(p-1)}\left(\Omega^{R}\right),\left|v_{x_{i}}\right|^{\frac{p-2}{2}} v_{x_{i} x_{i}} \in L^{2}\left(\Omega^{R}\right)\right\} \quad \text { for } i=1, \ldots, n
\end{aligned}
$$

and $H_{0}^{2}\left(\Omega^{R}\right)=\left\{u: u \in H^{2}\left(\Omega^{R}\right):\left.u\right|_{\partial \Omega^{R}}=0,\left.\frac{\partial u}{\partial \nu}\right|_{\partial \Omega^{R}}=0\right\}$. Moreover, let $l \in \mathbb{R}$ be at least such, that $V\left(\Omega^{R}\right) \subset H^{l}\left(\Omega^{R}\right)$ and $l>\frac{n+4}{2}$.

First, we consider the following equation

$$
A(u) \equiv f^{R}(x, t)
$$

in the domain $Q_{T}^{R}=\Omega^{R} \times(0, T), R>1$. For this equation we put the following boundary and initial conditions

$$
\begin{aligned}
& \left.u\right|_{t=0}=u_{0}^{R}(x),\left.\quad u_{t}\right|_{t=0}=u_{1}^{R}(x), \\
& \left.u\right|_{\partial \Omega^{R}}=\left.\frac{\partial u}{\partial \nu}\right|_{\partial \Omega^{R}}=0 .
\end{aligned}
$$

Definition 2.2. We call the function $u$ a solution of the problem (2.1)-(2.3) if

$$
\begin{aligned}
& u \in L^{2}\left((0, T) ; H_{0}^{2}\left(\Omega^{R}\right)\right), \quad u_{t t} \in L^{2}\left(Q_{T}^{R}\right), \\
& u_{t} \in L^{p}\left((0, T) ; W_{0}^{1, p}\left(\Omega^{R}\right)\right) \cap L^{q}\left(Q_{T}^{R}\right) \cap L^{2}\left((0, T) ; H_{0}^{2}\left(\Omega^{R}\right)\right)
\end{aligned}
$$

and $u$ satisfies the following integral equation

$$
\begin{aligned}
\int_{Q_{\tau}^{R}} & {\left[u_{t t} v+\sum_{i, j, k, l=1}^{n} a_{i j}^{k l}(x) u_{x_{i} x_{j}} v_{x_{k} x_{l}}+\sum_{i, j=1}^{n} a_{i j}(x) u_{x_{i}} v_{x_{j}}+a_{0}(x) u v\right.} \\
& \left.+\sum_{i=1}^{n} b_{i}(x)\left|u_{x_{i} t}\right|^{p-2} u_{x_{i} t} v_{x_{i}}+c(x)\left|u_{t}\right|^{q-2} u_{t} v\right] d x d t=\int_{Q_{\tau}^{R}} f^{R}(x, t) v d x d t
\end{aligned}
$$

$\forall v \in L^{2}\left((0, T) ; H_{0}^{2}\left(\Omega^{R}\right)\right) \cap L^{q}\left(Q_{T}^{R}\right) \cap L^{p}\left((0, T) ; W_{0}^{1, p}\left(\Omega^{R}\right)\right), \forall \tau \in(0, T]$. Moreover, $u$ satisfies the conditions $(2.2)$.

Theorem 2.3. If the conditions $\left(\mathbf{A}_{\mathbf{1}}\right)-\left(\mathbf{A}_{\mathbf{3}}\right),(\mathbf{B}),(\mathbf{C})$ hold and $f^{R}, f_{t}^{R} \in L^{2}\left(Q_{T}^{R}\right)$, $u_{0}^{R} \in H_{0}^{2}\left(\Omega^{R}\right) \cap H^{4}\left(\Omega^{R}\right), u_{1}^{R} \in V\left(\Omega^{R}\right) \cap H_{0}^{2}\left(\Omega^{R}\right)$ then there exist a solution of the problem (2.1)-(2.3).

Proof. Consider the space $H_{0}^{m}\left(\Omega^{R}\right)$, where $m=\max \{4, l\}$ and $\left\{\phi_{k}(x)\right\}$ is the basis of this space and $\left\{\phi_{k}(x)\right\}$ is orthonormal basis in $L^{2}\left(\Omega^{R}\right)$. Next, consider the sequence of functions of the form

$$
u^{N}(x, t)=\sum_{s=1}^{N} C_{s}^{N}(t) \phi_{s}(x)
$$


for $N=1,2, \ldots$, where the functions $C_{1}^{N}, \ldots, C_{N}^{N}$ are the solution of the following Cauchy problem:

$$
\begin{aligned}
& \int_{\Omega^{R}}\left[u_{t t}^{N}(x, t) \phi_{s}(x)+\sum_{i, j, k, l=1}^{n} a_{i j}^{k l}(x) u_{x_{i} x_{j}}^{N}(x, t) \phi_{s x_{k} x_{l}}(x)\right. \\
& \quad+\sum_{i, j=1}^{n} a_{i j}(x) u_{x_{i}}^{N}(x, t) \phi_{s x_{j}}(x)+\sum_{i=1}^{n} b_{i}(x)\left|u_{t x_{i}}^{N}(x, t)\right|^{p-2} u_{t x_{i}}^{N}(x, t) \phi_{s x_{i}}(x) \\
& \quad+a_{0}(x) u^{N}(x, t) \phi_{s}(x)+c(x)\left|u_{t}^{N}(x, t)\right|^{q-2} u_{t}^{N}(x, t) \phi_{s}(x) \\
& \left.\quad-f(x, t) \phi_{s}(x)\right] d x=0
\end{aligned}
$$

with the conditions

$$
C_{s}^{N}(0)=u_{0, s}^{N}, \quad C_{s t}^{N}(0)=u_{1, s}^{N}, \quad s=1, \ldots, N
$$

and $\left\|u_{0}^{N}-u_{0}\right\|_{H_{0}^{2}\left(\Omega^{R}\right) \cap H^{4}\left(\Omega^{R}\right)} \rightarrow 0,\left\|u_{1}^{N}-u_{1}\right\|_{H_{0}^{2}\left(\Omega^{R}\right)} \rightarrow 0$, where $u_{0}^{N}(x)=$ $\sum_{s=1}^{N} u_{0, s}^{N} \phi_{s}(x)$ and $u_{1}^{N}(x)=\sum_{s=1}^{N} u_{1, s}^{N} \phi_{s}(x)$.

From the Carathéodory theorem there exists the solution of problem (2.4)-(2.5), where $C_{s}^{N}$ are continuous and $C_{s t}^{N}, s=1, \ldots, N$ are absolutely continuous on the interval $\left[0, h_{0}\right]$. Taking into account the estimations obtained below we can state that $h_{0}=T$.

Multiplying (2.4) by the functions $C_{s t}^{N}(t) e^{-\eta t}, \eta>0$ respectively, then summing by $s$ from 1 to $N$ and integrating with respect to $t$ from 0 to $\tau, \tau \in(0, T]$, we obtain

$$
\begin{aligned}
& \int_{Q_{\tau}^{R}}\left[u_{t t}^{N} u_{t}^{N} e^{-\eta t}+\sum_{i, j, k, l=1}^{n} a_{i j}^{k l}(x) u_{x_{i} x_{j}}^{N} u_{t x_{k} x_{l}}^{N} e^{-\eta t}+\sum_{i, j=1}^{n} a_{i j}(x) u_{x_{i}}^{N} u_{t x_{j}}^{N} e^{-\eta t}\right. \\
& \quad+\sum_{i=1}^{n} b_{i}(x)\left|u_{t x_{i}}^{N}\right|^{p-2} u_{t x_{i}}^{N} u_{t x_{i}}^{N} e^{-\eta t}+a_{0}(x) u^{N} u_{t}^{N} e^{-\eta t} \\
& \left.\quad+c(x)\left|u_{t}^{N}\right|^{q-2} u_{t}^{N} u_{t}^{N} e^{-\eta t}-f(x, t) u_{t}^{N} e^{-\eta t}\right] d x d t=0
\end{aligned}
$$

If we consider the respective components of the last equality we will have

$$
\int_{Q_{\tau}^{R}} u_{t t}^{N} u_{t}^{N} e^{-\eta t} d x d t=\frac{1}{2} \int_{\Omega_{\tau}^{R}}\left|u_{t}^{N}\right|^{2} e^{-\eta \tau} d x-\frac{1}{2} \int_{\Omega_{0}^{R}}\left|u_{1}^{N}\right|^{2} d x+\frac{\eta}{2} \int_{Q_{\tau}^{R}}\left|u_{t}^{N}\right|^{2} e^{-\eta t} d x d t
$$


where $\Omega_{\tau}^{R}=Q_{T}^{R} \cap\{t=\tau\}$. Next, from $\left(\mathbf{A}_{\mathbf{1}}\right)$ we have

$$
\begin{aligned}
& \int_{Q_{\tau}^{R}} \sum_{i, j, k, l=1}^{n} a_{i j}^{k l}(x) u_{x_{i} x_{j}}^{N} u_{t x_{k} x_{l}}^{N} e^{-\eta t} d x d t \geq \frac{a_{2}}{2} \int_{\Omega_{\tau}^{R}} \sum_{i, j=1}^{n}\left|u_{x_{i} x_{j}}^{N}\right|^{2} e^{-\eta \tau} d x \\
& -\frac{1}{2} \int_{\Omega_{0}^{R}} \sum_{i, j, k, l=1}^{n} a_{i j}^{k l}(x) u_{0 x_{i} x_{j}}^{N} u_{0 x_{k} x_{l}}^{N} d x+\frac{\eta a_{2}}{2} \int_{Q_{\tau}^{R}} \sum_{i, j=1}^{n}\left|u_{x_{i} x_{j}}^{N}\right|^{2} e^{-\eta t} d x d t .
\end{aligned}
$$

From the assumption $\left(\mathbf{A}_{\mathbf{2}}\right)$ and the initial condition we obtain

$$
\begin{aligned}
\int_{Q_{\tau}^{R}} & \sum_{i, j=1}^{n} a_{i j}(x) u_{x_{i}}^{N} u_{t x_{j}}^{N} e^{-\eta t} d x d t=\frac{1}{2} \int_{\Omega_{\tau}^{R}} \sum_{i, j=1}^{n} a_{i j}(x) u_{x_{i}}^{N} u_{x_{j}}^{N} e^{-\eta \tau} d x \\
& -\frac{1}{2} \int_{\Omega_{0}^{R}} \sum_{i, j=1}^{n} a_{i j}(x) u_{0 x_{i}}^{N} u_{0 x_{j}}^{N} d x+\frac{\eta}{2} \int_{Q_{\tau}^{R}} \sum_{i, j=1}^{n} a_{i j}(x) u_{x_{i}}^{N} u_{x_{j}}^{N} e^{-\eta t} d x d t \\
\geq & \frac{a_{1}}{2} \int_{\Omega_{\tau}^{R}} \sum_{i=1}^{n}\left|u_{x_{i}}^{N}\right|^{2} e^{-\eta \tau} d x+\frac{a_{1} \eta}{2} \int_{Q_{\tau}^{R}} \sum_{i=1}^{n}\left|u_{x_{i}}^{N}\right|^{2} e^{-\eta t} d x d t \\
& -\frac{1}{2} \int_{\Omega_{0}^{R}} \sum_{i, j=1}^{n} a_{i j}(x) u_{0 x_{i}}^{N} u_{0 x_{j}}^{N} d x .
\end{aligned}
$$

Next, from the assumption (B) we have

$$
\int_{Q_{\tau}^{R}} \sum_{i=1}^{n} b_{i}(x)\left|u_{t x_{i}}^{N}\right|^{p-2} u_{t x_{i}}^{N} u_{t x_{i}}^{N} e^{-\eta t} d x d t \geq b_{0} \int_{Q_{\tau}^{R}} \sum_{i=1}^{n}\left|u_{t x_{i}}^{N}\right|^{p} e^{-\eta t} d x d t .
$$

From $\left(\mathbf{A}_{\mathbf{3}}\right)$ we obtain

$$
\begin{aligned}
& \int_{Q_{\tau}^{R}} a_{0}(x) u^{N} u_{t}^{N} e^{-\eta t} d x d t=\frac{1}{2} \int_{\Omega_{\tau}^{R}} a_{0}\left|u^{N}\right|^{2} e^{-\eta \tau} d x \\
& \quad-\frac{1}{2} \int_{\Omega_{0}^{R}} a_{0}(x)\left|u_{0}^{N}\right|^{2} d x+\frac{\eta}{2} \int_{Q_{\tau}^{R}} a_{0}(x)\left|u^{N}\right|^{2} e^{-\eta t} d x d t \geq-\frac{1}{2} \int_{\Omega_{0}^{R}} a_{0}(x)\left|u_{0}^{N}\right|^{2} d x .
\end{aligned}
$$

From (C) we have

$$
\int_{Q_{\tau}^{R}} c(x)\left|u_{t}^{N}\right|^{q-2} u_{t}^{N} u_{t}^{N} e^{-\eta t} d x d t \geq c_{0} \int_{Q_{\tau}^{R}}\left|u_{t}^{N}\right|^{q} e^{-\eta t} d x d t
$$

Next,

$$
\int_{Q_{\tau}^{R}} f(x, t) u_{t}^{N} e^{-\eta t} d x d t \leq \frac{1}{2} \int_{Q_{\tau}^{R}}|f(x, t)|^{2} e^{-\eta t} d x d t+\frac{1}{2} \int_{Q_{\tau}^{R}}\left|u_{t}^{N}\right|^{2} e^{-\eta t} d x d t .
$$


Thus, from (2.6) we get the following inequality

$$
\begin{aligned}
\int_{\Omega_{\tau}^{R}} & {\left[\left|u_{t}^{N}\right|^{2}+a_{2} \sum_{i, j=1}^{n}\left|u_{x_{i} x_{j}}^{N}\right|^{2}+a_{1} \sum_{i=1}^{n}\left|u_{x_{i}}^{N}\right|^{2}\right] e^{-\eta \tau} d x+\int_{Q_{\tau}^{R}}\left[(\eta-1)\left|u_{t}^{N}\right|^{2}\right.} \\
& \left.+c_{0}\left|u_{t}^{N}\right|^{q}+\eta a_{2} \sum_{i, j=1}^{n}\left|u_{x_{i} x_{j}}^{N}\right|^{2}+\eta a_{1} \sum_{i=1}^{n}\left|u_{x_{i}}^{N}\right|^{2}+b_{0} \sum_{i=1}^{n}\left|u_{t x_{i}}^{N}\right|^{p}\right] e^{-\eta t} d x d t \\
\leq & \int_{\Omega_{0}^{R}}\left[\left|u_{1}^{N}\right|^{2}+\sum_{i, j, k, l=1}^{n} a_{i j}^{k l}(x) u_{0 x_{i} x_{j}}^{N} u_{0 x_{k} x_{l}}^{N}\right. \\
& \left.+\sum_{i, j=1}^{n} a_{i j}(x) u_{0 x_{i}}^{N} u_{0 x_{j}}^{N}+a_{0}(x)\left|u_{0}^{N}\right|^{2}\right] d x+\int_{Q_{\tau}^{R}}|f(x, t)|^{2} e^{-\eta t} d x d t .
\end{aligned}
$$

Due to the assumptions of Theorem 2.3 we can differentiate the system (2.4) with respect to $t$

$$
\begin{aligned}
& \int_{\Omega^{R}}\left[u_{t t t}^{N}(x, t) \phi_{s}(x)+\sum_{i, j, k, l=1}^{n} a_{i j}^{k l}(x) u_{t x_{i} x_{j}}^{N}(x, t) \phi_{s x_{k} x_{l}}(x)\right. \\
& \quad+\sum_{i, j=1}^{n} a_{i j}(x) u_{t x_{i}}^{N}(x, t) \phi_{s x_{j}}(x) \\
& \quad+\sum_{i=1}^{n} b_{i}(x)\left(\left|u_{t x_{i}}^{N}(x, t)\right|^{p-2} u_{t x_{i}}^{N}(x, t)\right)_{t} \phi_{s x_{i}}(x)+a_{0}(x) u_{t}^{N}(x, t) \phi_{s}(x) \\
& \left.\quad+c(x)\left(\left|u_{t}^{N}(x, t)\right|^{q-2} u_{t}^{N}(x, t)\right)_{t} \phi_{s}(x)-f_{t}(x, t) \phi_{s}(x)\right] d x=0 .
\end{aligned}
$$

Multiplying (2.8) by the functions $C_{s t t}^{N}(t) e^{-\eta t}$, respectively, then summing by $s$ from 1 to $N$ and integrating with respect to $t$ from 0 to $\tau, \tau \in(0, T]$, we obtain

$$
\begin{aligned}
& \int_{Q_{\tau}^{R}}\left[u_{t t t}^{N} u_{t t}^{N} e^{-\eta t}+\sum_{i, j, k, l=1}^{n} a_{i j}^{k l}(x) u_{t x_{i} x_{j}}^{N} u_{t t x_{k} x_{l}}^{N} e^{-\eta t}\right. \\
& \quad+\sum_{i, j=1}^{n} a_{i j}(x) u_{t x_{i}}^{N} u_{t t x_{j}}^{N} e^{-\eta t}+\sum_{i=1}^{n} b_{i}(x)\left(\left|u_{t x_{i}}^{N}\right|^{p-2} u_{t x_{i}}^{N}\right)_{t} u_{t t x_{i}}^{N} e^{-\eta t} \\
& \left.\quad+a_{0}(x) u_{t}^{N} u_{t t}^{N} e^{-\eta t}+c(x)\left(\left|u_{t}^{N}\right|^{q-2} u_{t}^{N}\right)_{t} u_{t t}^{N} e^{-\eta t}-f_{t}(x, t) u_{t t}^{N} e^{-\eta t}\right] d x d t=0 .
\end{aligned}
$$


Observe, that for $t=0$ multiplying (2.8) by $C_{s t t}(0)$, by analogy to $(2.9)$, we obtain

$$
\begin{aligned}
\int_{\Omega_{0}^{R}} & {\left[\left|u_{t t}^{N}\right|^{2}(x, 0)+\sum_{i, j, k, l=1}^{n}\left(a_{i j}^{k l}(x) u_{x_{i} x_{j}}^{N}(x, 0)\right)_{x_{k} x_{l}} u_{t t}^{N}(x, 0)\right.} \\
& -\sum_{i, j=1}^{n}\left(a_{i j}(x) u_{x_{i}}^{N}(x, 0)\right)_{x_{j}} u_{t t}^{N}(x, 0) \\
& -\sum_{i=1}^{n}\left(b_{i}(x)\left|u_{t x_{i}}^{N}(x, 0)\right|^{p-2} u_{t x_{i}}^{N}(x, 0)\right)_{x_{i}} u_{t t}^{N}(x, 0) \\
& +a_{0}(x) u^{N}(x, 0) u_{t t}^{N}(x, 0) \\
& \left.+c(x)\left|u_{t}^{N}(x, 0)\right|^{q-2} u_{t}^{N}(x, 0) u_{t t}^{N}(x, 0)-f(x, 0) u_{t t}^{N}(x, 0)\right] d x=0 .
\end{aligned}
$$

It is easy to prove that for all $u \in H_{0}^{l}\left(\Omega^{R}\right)$,

$$
\int_{\Omega^{R}}\left(\left|u_{x_{i}}\right|^{p-2} u_{x_{i} x_{i}}\right)^{2} d x \leq K\left(\int_{\Omega^{R}}\left|u_{x_{i} x_{i}}\right|^{2} d x\right)^{p-1}
$$

where $K$ depends on $R$. Then by conditions of Theorem 2.3

$$
\begin{aligned}
\int_{\Omega_{0}^{R}} & {\left[\left|\sum_{i, j, k, l=1}^{n}\left(a_{i j}^{k l} u_{0 x_{i} x_{j}}^{N}\right)_{x_{k} x_{l}}\right|^{2}+\left|\sum_{i, j=1}^{n}\left(a_{i j} u_{0 x_{i}}^{N}\right)_{x_{j}}\right|^{2}\right.} \\
+ & \left.\left|\sum_{i=1}^{n}\left(b_{i}(x)\left|u_{1 x_{i}}^{N}\right|^{p-2} u_{1 x_{i}}^{N}\right)_{x_{i}}\right|^{2}+\left|a_{0} u_{0}^{N}\right|^{2}+\left(c(x)\left|u_{1}^{N}\right|^{q-2} u_{1}^{N}\right)\right] d x \leq K_{1}
\end{aligned}
$$

and from (2.10) it follows then

$$
\int_{\Omega_{0}^{R}}\left|u_{t t}^{N}\right|^{2} d x \leq M_{1}
$$

where $M_{1}$ does not depend on $N$. Returning to (2.9), we have from (2.11)

$$
\begin{aligned}
I_{1}:= & \int_{Q_{\tau}^{R}} u_{t t t}^{N} u_{t t}^{N} e^{-\eta t} d x d t=\frac{1}{2} \int_{\Omega_{\tau}^{R}}\left|u_{t t}^{N}\right|^{2} e^{-\eta \tau} d x-\frac{1}{2} \int_{\Omega_{0}^{R}}\left|u_{t t}^{N}\right|^{2} d x \\
& +\frac{\eta}{2} \int_{Q_{\tau}^{R}}\left|u_{t t}^{N}\right|^{2} e^{-\eta t} d x d t \geq \frac{1}{2} \int_{\Omega_{\tau}^{R}}\left|u_{t t}^{N}\right|^{2} e^{-\eta \tau} d x+\frac{\eta}{2} \int_{Q_{\tau}^{R}}\left|u_{t t}^{N}\right|^{2} e^{-\eta t} d x d t-\frac{M_{1}}{2} .
\end{aligned}
$$


From the assumption $\left(\mathbf{A}_{\mathbf{1}}\right)$, we have

$$
\begin{aligned}
I_{2}:= & \int_{Q_{\tau}^{R}} \sum_{i, j, k, l=1}^{n} a_{i j}^{k l}(x) u_{t x_{i} x_{j}}^{N} u_{t x_{k} x_{l} t}^{N} e^{-\eta t} d x d t \\
\geq & \frac{a_{2}}{2} \int_{\Omega_{\tau}^{R}} \sum_{i, j=1}^{n}\left|u_{t x_{i} x_{j}}^{N}\right|^{2} e^{-\eta \tau} d x \\
& -\frac{1}{2} \int_{\Omega_{0}^{R}} \sum_{i, j, k, l=1}^{n} a_{i j}^{k l}(x) u_{1 x_{i} x_{j}}^{N} u_{1 x_{k} x_{l}}^{N} d x+\frac{\eta a_{2}}{2} \int_{Q_{\tau}^{R}} \sum_{i, j=1}^{n}\left|u_{t x_{i} x_{j}}^{N}\right|^{2} e^{-\eta t} d x d t .
\end{aligned}
$$

From the assumption $\left(\mathbf{A}_{\mathbf{2}}\right)$ and the initial condition we obtain

$$
\begin{aligned}
I_{3}:= & \int_{Q_{\tau}^{R}} \sum_{i, j=1}^{n} a_{i j}(x) u_{t x_{i}}^{N} u_{t x_{j} t}^{N} e^{-\eta t} d x d t \geq \frac{a_{1}}{2} \int_{\Omega_{\tau}^{R}} \sum_{i=1}^{n}\left|u_{t x_{i}}^{N}\right|^{2} e^{-\eta \tau} d x \\
& +\frac{a_{1} \eta}{2} \int_{Q_{\tau}^{R}} \sum_{i=1}^{n}\left|u_{t x_{i}}^{N}\right|^{2} e^{-\eta t} d x d t-\frac{1}{2} \int_{\Omega_{0}^{R}} \sum_{i, j=1}^{n} a_{i j}(x) u_{1 x_{i}}^{N} u_{1 x_{j}}^{N} d x .
\end{aligned}
$$

Next, from the assumption (B) we have

$$
\begin{aligned}
I_{4} & :=\int_{Q_{\tau}^{R}} \sum_{i=1}^{n} b_{i}(x)\left(\left|u_{t x_{i}}^{N}\right|^{p-2} u_{t x_{i}}^{N}\right)_{t} u_{t t x_{i}}^{N} e^{-\eta t} d x d t \\
& \geq b_{0}(p-1) \int_{Q_{\tau}^{R}} \sum_{i=1}^{n}\left|u_{t x_{i}}^{N}\right|^{p} e^{-\eta t} d x d t \\
I_{5} & :=\int_{Q_{\tau}^{R}} a_{0}(x) u_{t}^{N} u_{t t}^{N} e^{-\eta t} d x d t \leq \frac{a_{0}^{1}}{2} \int_{Q_{\tau}^{R}}\left|u_{t}^{N}\right|^{2} e^{-\eta t} d x d t+\frac{a_{0}^{1}}{2} \int_{Q_{\tau}^{R}}\left|u_{t t}^{N}\right|^{2} e^{-\eta t} d x d t
\end{aligned}
$$

where $a_{0}^{1}=\operatorname{ess} \sup _{\Omega} a_{0}(x)$. From $(\mathbf{C})$ we have

$$
\begin{aligned}
I_{6} & :=\int_{Q_{\tau}^{R}} c(x)\left(\left|u_{t}^{N}\right|^{q-2} u_{t}^{N}\right)_{t} u_{t t}^{N} e^{-\eta t} d x d t \\
& \geq c_{0} \int_{Q_{\tau}^{R}}(q-1)\left|u_{t}^{N}\right|^{q-2}\left|u_{t t}^{N}\right|^{2} e^{-\eta t} d x d t \geq 0 . \\
I_{7} & :=\int_{Q_{\tau}^{R}} f_{t}(x, t) u_{t t}^{N} e^{-\eta t} d x d t \leq \frac{1}{2} \int_{Q_{\tau}^{R}}\left|f_{t}(x, t)\right|^{2} e^{-\eta t} d x d t+\frac{1}{2} \int_{Q_{\tau}^{R}}\left|u_{t t}^{N}\right|^{2} e^{-\eta t} d x d t .
\end{aligned}
$$


Thus, from (2.9), taking into account the estimates of the integrals $I_{1}-I_{7}$, we get the following inequality

$$
\begin{aligned}
& \int_{\Omega_{\tau}^{R}}\left[\left|u_{t t}^{N}\right|^{2}+a_{2} \sum_{i, j=1}^{n}\left|u_{t x_{i} x_{j}}^{N}\right|^{2}+a_{1} \sum_{i=1}^{n}\left|u_{t x_{i}}^{N}\right|^{2}\right] e^{-\eta \tau} d x+\int_{Q_{\tau}^{R}}\left[\left(\eta-a_{0}^{1}-1\right)\left|u_{t t}^{N}\right|^{2}\right. \\
& \quad+\eta a_{1} \sum_{i=1}^{n}\left|u_{t x_{i}}^{N}\right|^{2}+2 b_{0}(p-1) \sum_{i=1}^{n}\left|u_{t x_{i}}^{N}\right|^{p}+2 c_{0}(q-1)\left|u_{t}^{N}\right|^{q-2}\left|u_{t t}^{N}\right|^{2} \\
& \left.\quad+\eta a_{2} \sum_{i, j=1}^{n}\left|u_{t x_{i} x_{j}}^{N}\right|^{2}\right] e^{-\eta t} d x d t \leq \int_{Q_{\tau}^{R}}\left|f_{t}(x, t)\right|^{2} e^{-\eta t} d x d t \\
& \quad+\int_{\Omega_{0}^{R}}\left[\sum_{i, j=1}^{n} a_{i j}(x) u_{1 x_{i}}^{N} u_{1 x_{j}}^{N}+\sum_{i, j, k, l=1}^{n} a_{i j}^{k l}(x) u_{1 x_{i} x_{j}}^{N} u_{1 x_{k} x_{l}}^{N}\right] d x+M_{1} \leq M_{2} .
\end{aligned}
$$

From (2.7) and (2.12), choosing $\eta=a_{0}^{1}+2$, we obtain that $\left\{u^{N}\right\}$ is bounded in the space $L^{\infty}\left((0, T) ; H_{0}^{2}\left(\Omega^{R}\right)\right)$ and $\left\{u_{t t}^{N}\right\}$ is bounded in $L^{\infty}\left((0, T) ; L^{2}\left(\Omega^{R}\right)\right)$, and $\left\{u_{t}^{N}\right\}$ is bounded in $L^{p}\left((0, T) ; W_{0}^{1, p}\left(\Omega^{R}\right)\right) \cap L^{q}\left(Q_{T}^{R}\right) \cap L^{\infty}\left((0, T) ; H_{0}^{2}\left(\Omega^{R}\right)\right)$. Hence, we can choose subsequences $\left\{u^{N_{k}}\right\},\left\{u_{t}^{N_{k}}\right\},\left\{u_{t t}^{N_{k}}\right\}$ such that $u^{N_{k}} \rightarrow u^{R *}$-weakly in $L^{\infty}\left((0, T) ; H_{0}^{2}\left(\Omega^{R}\right)\right), u_{t t}^{N_{k}} \rightarrow u_{t t}^{R}{ }^{*}$-weakly in $L^{\infty}\left((0, T) ; L^{2}\left(\Omega^{R}\right)\right), u_{t}^{N_{k}} \rightarrow u_{t}^{R}$ *-weakly in $L^{\infty}\left((0, T) ; H_{0}^{2}\left(\Omega^{R}\right)\right), u_{t}^{N_{k}} \rightarrow u_{t}^{R}$ weakly in $L^{p}\left((0, T) ; W_{0}^{1, p}\left(\Omega^{R}\right)\right) \cap L^{q}$ $\left(Q_{T}^{R}\right)$ as $N_{k} \rightarrow \infty$. Define the operator $B: L^{p}\left((0, T) ; W_{0}^{1, p}\left(\Omega^{R}\right)\right) \cap L^{q}\left(Q_{T}^{R}\right) \rightarrow$ $L^{p^{\prime}}\left((0, T) ; W_{0}^{-1, p}\left(\Omega^{R}\right)\right)+L^{q^{\prime}}\left(Q_{T}^{R}\right)$ by the formula

$$
\langle B(u), v\rangle=\int_{Q_{T}^{R}}\left[\sum_{i=1}^{n} b_{i}\left|u_{x_{i}}\right|^{p-2} u_{x_{i}} v_{x_{i}}+c|u|^{q-2} u\right] d x d t
$$

for $u, v \in L^{p}\left((0, T) ; W_{0}^{1, p}\left(\Omega^{R}\right)\right) \cap L^{q}\left(Q_{T}^{R}\right)$. It is easy to see that $B$ is monotonic, and $\left\{B\left(u_{t}^{N}\right)\right\}$ is bounded in the space $L^{p^{\prime}}\left((0, T) ; W_{0}^{-1, p^{\prime}}\left(\Omega^{R}\right)\right)+L^{q^{\prime}}\left(Q_{T}^{R}\right)$. Hence, we can accept that $B\left(u_{t}^{N_{k}}\right) \rightarrow z$ weakly in $L^{p^{\prime}}\left((0, T) ; W_{0}^{-1, p}\left(\Omega^{R}\right)\right)+L^{q^{\prime}}\left(Q_{T}^{R}\right)$. Analogously as in $\left[20\right.$, p.II,s.1,2] we can prove that $z=B\left(u_{t}^{R}\right)$. Since $u_{t t}^{R} \in$ $L^{\infty}\left((0, T) ; L^{2}\left(\Omega^{R}\right)\right)$, and $u_{t} \in L^{\infty}\left((0, T) ; H^{2}\left(\Omega^{R}\right)\right)$, then $u_{t} \in C\left([0, T] ; L^{2}\left(\Omega^{R}\right)\right)$. Analogously, $u \in C\left([0, T] ; H_{0}^{2}\left(\Omega^{R}\right)\right)$. By analogy with $[20$, p.I, s.1,4] it is easy to prove that $\left.u^{R}\right|_{t=0}=u_{0}^{R}(x),\left.u_{t}^{R}\right|_{t=0}=u_{1}^{R}(x)$. Hence the function $u^{R}$ is the solution of the problem (2.1)-(2.3) in the sense of Definition 2.2.

Theorem 2.4. If the conditions $\left(\mathbf{A}_{\mathbf{1}}\right)-\left(\mathbf{A}_{\mathbf{3}}\right),(\mathbf{B}),(\mathbf{C})$ hold, and $f, f_{t} \in L_{l o c}^{2}\left(Q_{T}\right)$, $\frac{2 n}{n-2}>q>p>2, u_{0} \in H_{0, \mathrm{loc}}^{2}(\bar{\Omega}), u_{1} \in V_{\text {loc }}(\bar{\Omega})$, then the problem $(1.1)-(1.3)$ has a weak solution. 
Proof. Let $f^{s}(x, t)=\left\{\begin{array}{ll}f(x, t), & \text { for }(x, t) \in Q_{T}^{s}, \\ 0, & \text { for }(x, t) \in Q_{T} \backslash Q_{T}^{s}, s=2,3,4 \ldots\end{array}\right.$ and $u_{0, s}(x)=$ $u_{0}(x) \theta(x), \quad u_{1, s}(x)=u_{1}(x) \theta(x)$, where $\theta \in C^{4}\left(\mathbb{R}^{n}\right), 0 \leq \theta(x) \leq 1$, and $\theta(x)=$ $\left\{\begin{array}{l}1, \text { for }|x| \leq s-1 \\ 0, \text { for }|x| \geq s .\end{array}\right.$ We shall consider in the domain $Q_{T}^{s}$ the equation

$$
A(u)=f^{s}(x, t)
$$

with the following boundary and initial conditions

$$
\begin{aligned}
& \left.u\right|_{S_{T}^{s}}=0,\left.\quad \frac{\partial u}{\partial \nu}\right|_{S_{T}^{s}}=0, \\
& \left.u\right|_{t=0}=u_{0, s}(x),\left.\quad u_{t}\right|_{t=0}=u_{1, s}(x),
\end{aligned}
$$

where $S_{T}^{s}=\partial \Omega^{s} \times(0, T)$. Due to Theorem 2.3 there is the solution $u^{s}$ of the problem (2.13)-(2.15). If we extend this solution by 0 at the set $Q_{T} \backslash Q_{T}^{s}$ then for $\tau \in(0, T]$ we obtain

$$
\begin{aligned}
& \int_{Q_{\tau}}\left[u_{t t}^{s} v+\sum_{i, j, k, l=1}^{n} a_{i j}^{k l}(x) u_{x_{i} x_{j}}^{s} v_{x_{k} x_{l}}+\sum_{i, j=1}^{n} a_{i j}(x) u_{x_{i}}^{s} v_{x_{j}}\right. \\
& \quad+\sum_{i=1}^{n} b_{i}(x)\left|u_{t x_{i}}^{s}\right|^{p-2} u_{t x_{i}}^{s} v_{x_{i}}+a_{0}(x) u^{s} v \\
& \left.+c(x)\left|u_{t}^{s}\right|^{q-2} u_{t}^{s} v-f^{s}(x, t) v\right] d x d t=0
\end{aligned}
$$

for all $v$ such that $v \in L^{2}\left((0, T) ; H_{0, \text { loc }}^{2}(\bar{\Omega})\right), v_{t} \in L^{p}\left((0, T) ; W_{0, \text { loc }}^{1, p}(\bar{\Omega})\right) \cap L^{q}$ $\left((0, T) ; L_{\text {loc }}^{q}(\bar{\Omega})\right)$. The function $u^{s}$ satisfies the initial conditions

$$
u^{s}(x, 0)=u_{0, s}(x), \quad u_{t}^{s}(x, 0)=u_{1, s}(x), \quad x \in \Omega .
$$

For the fixed $R>1$ we choose $v(x, t)=u_{t}^{s}(x, t) \Psi_{R}(x) e^{-\eta t}$, where

$$
\Psi_{R}(x)=\left[h_{R}(x)\right]^{\beta}, \beta>1,
$$

and

$$
h_{R}(x)= \begin{cases}\frac{1}{R}\left(R^{2}-|x|^{2}\right), & \text { for } 0 \leq|x| \leq R \\ 0, & \text { for }|x|>R\end{cases}
$$

From (2.17) we have

$$
\Psi_{R x_{i}}=\beta\left(h_{R}\right)^{\beta-1} h_{R x_{i}}, \quad h_{R x_{i}}=-\frac{2 x_{i}}{R}, \quad\left|h_{R x_{i}}\right| \leq 2, \text { for } i=1,2, \ldots, n .
$$


If we consider the respective components of the equality (2.16) we will have

$$
\begin{aligned}
I_{8}:= & \int_{Q_{\tau}} u_{t t}^{s} u_{t}^{s} \Psi_{R}(x) e^{-\eta t} d x d t=\frac{1}{2} \int_{\Omega_{\tau}}\left|u_{t}^{s}\right|^{2} \Psi_{R}(x) e^{-\eta \tau} d x \\
& -\frac{1}{2} \int_{\Omega_{0}}\left|u_{1, s}^{s}\right|^{2} \Psi_{R}(x) d x+\frac{\eta}{2} \int_{Q_{\tau}}\left|u_{t}^{s}\right|^{2} \Psi_{R}(x) e^{-\eta t} d x d t .
\end{aligned}
$$

From $\left(\mathbf{A}_{\mathbf{1}}\right)$ we have

$$
\begin{aligned}
I_{9}:= & \int_{Q_{\tau}} \sum_{i, j, k, l=1}^{n} a_{i j}^{k l}(x) u_{x_{i} x_{j}}^{s} v_{x_{k} x_{l}} d x d t=I_{9}^{1}+I_{9}^{2}+I_{9}^{3}, \text { where } \\
I_{9}^{1}:= & \int_{Q_{\tau}} \sum_{i, j, k, l=1}^{n} a_{i j}^{k l}(x) u_{x_{i} x_{j}}^{s} u_{t x_{k} x_{l}}^{s} \Psi_{R}(x) e^{-\eta t} d x d t \\
\geq & \frac{a_{2}}{2} \int_{\Omega_{\tau}} \sum_{i, j=1}^{n}\left|u_{x_{i} x_{j}}^{s}\right|^{2} \Psi_{R}(x) e^{-\eta \tau} d x \\
& -\frac{1}{2} \int_{\Omega_{0}} \sum_{i, j, k, l=1}^{n} a_{i j}^{k l}(x) u_{0, s x_{i} x_{j}}^{s} u_{0, s x_{k} x_{l}}^{s} \Psi_{R}(x) d x \\
& +\frac{\eta a_{2}}{2} \int_{Q_{\tau}} \sum_{i, j=1}^{n}\left|u_{x_{i} x_{j}}^{s}\right|^{2} \Psi_{R}(x) e^{-\eta t} d x d t \quad \text { and } \\
I_{9}^{2}:= & \int_{Q_{\tau}} \sum_{i, j, k, l=1}^{n} a_{i j}^{k l}(x) u_{x_{i} x_{j}}^{s} u_{t x_{k}}^{s}\left[\Psi_{R x_{l}}(x)+\Psi_{R x_{k}}(x)\right] e^{-\eta t} d x d t, \\
I_{9}^{3}:= & \int_{Q_{\tau}} \sum_{i, j, k, l=1}^{n} a_{i j}^{k l}(x) u_{x_{i} x_{j}}^{s} u_{t}^{s} \Psi_{R x_{l}, x_{k}}(x) e^{-\eta t} d x d t .
\end{aligned}
$$

Using Hölder's and Young's inequalities we from (2.15) obtain

$$
\begin{aligned}
I_{9}^{2} \leq & \left(\int_{Q_{\tau}} \sum_{i, j, k, l=1}^{n}\left|a_{i j}^{k l}(x)\right|^{2}\left|u_{x_{i} x_{j}}^{s}\right|^{2} \Psi_{R}(x) e^{-\eta t} d x d t\right)^{\frac{1}{2}} \\
& \times\left(\int_{Q_{\tau}} \sum_{k=1}^{n}\left|u_{t x_{k}}^{s}\right|^{p} \Psi_{R}(x) e^{-\eta t} d x d t\right)^{\frac{1}{p}} \cdot\left(\int_{Q_{\tau}} \sum_{l=1}^{n}\left(\frac{\Psi_{R x_{l}}}{\Psi_{R}^{\frac{1}{2}+\frac{1}{p}}}\right)^{r} e^{-\eta t} d x d t\right)^{\frac{1}{r}} \\
\leq & \frac{\delta_{2}}{2} \int_{Q_{\tau}} \sum_{i, j, k, l=1}^{n}\left|a_{i j}^{k l}(x)\right|^{2}\left|u_{x_{i} x_{j}}^{s}\right|^{2} \Psi_{R}(x) e^{-\eta t} d x d t \\
& +\frac{\delta_{2}}{2 p} \int_{Q_{\tau}} \sum_{k=1}^{n}\left|u_{t x_{k}}^{s}\right|^{p} \Psi_{R}(x) e^{-\eta t} d x d t+\mu\left(\delta_{2}\right) \int_{Q_{\tau}} \sum_{l=1}^{n}\left(\frac{\Psi_{R x_{l}}}{\Psi_{R}^{\frac{1}{2}+\frac{1}{p}}}\right)^{r} e^{-\eta t} d x d t
\end{aligned}
$$




$$
\begin{aligned}
\leq & \frac{\delta_{2}}{2} a_{2}^{0} \int_{Q_{\tau}} \sum_{i, j=1}^{n}\left|u_{x_{i} x_{j}}^{s}\right|^{2} \Psi_{R}(x) e^{-\eta t} d x d t+\frac{\delta_{2}}{2 p} \int_{Q_{\tau}} \sum_{k=1}^{n}\left|u_{t x_{k}}^{s}\right|^{p} \Psi_{R}(x) e^{-\eta t} d x d t \\
& +\mu\left(\delta_{2}\right) \int_{Q_{\tau}} \sum_{l=1}^{n}\left(\frac{\Psi_{R x_{l}}}{\Psi_{R}^{\frac{1}{2}+\frac{1}{p}}}\right)^{r} e^{-\eta t} d x d t,
\end{aligned}
$$

where $r=\frac{2 p}{p-2}, \delta_{2}>0$. Similarly,

$$
\begin{aligned}
I_{9}^{3} \leq & \left(\int_{Q_{\tau}} \sum_{i, j, k, l=1}^{n}\left|a_{i j}^{k l}(x)\right|^{2}\left|u_{x_{i} x_{j}}^{s}\right|^{2} \Psi_{R}(x) e^{-\eta t} d x d t\right)^{\frac{1}{2}} \\
& \cdot\left(\int_{Q_{\tau}}\left|u_{t}^{s}\right|^{q} \Psi_{R}(x) e^{-\eta t} d x d t\right)^{\frac{1}{q}} \cdot\left(\int_{Q_{\tau}} \sum_{k, l=1}^{n}\left(\frac{\Psi_{R x_{l} x_{k}}}{\Psi_{R}^{\frac{1}{2}+\frac{1}{q}}}\right)^{r_{1}} e^{-\eta t} d x d t\right)^{\frac{1}{r_{1}}} \\
\leq & \frac{a_{2}^{0} \delta_{2}}{2} \int_{Q_{\tau}} \sum_{i, j=1}^{n}\left|u_{x_{i} x_{j}}^{s}\right|^{2} \Psi_{R}(x) e^{-\eta t} d x d t+\frac{\delta_{2}}{2 q} \int_{Q_{\tau}}\left|u_{t}^{s}\right|^{q} \Psi_{R}(x) e^{-\eta t} d x d t \\
& +\mu_{1}\left(\delta_{2}\right) \int_{Q_{\tau}} \sum_{k, l=1}^{n}\left(\frac{\Psi_{R x_{l} x_{k}}}{\Psi_{R}^{\frac{1}{2}+\frac{1}{q}}}\right)^{r_{1}} e^{-\eta t} d x d t
\end{aligned}
$$

where $r_{1}=\frac{2 q}{q-2}, a_{2}^{0}=\max _{i, j, k, l} \operatorname{ess} \sup _{\Omega}\left(a_{i j}^{k l}(x)\right)^{2}$.

From $\left(\mathbf{A}_{\mathbf{2}}\right)$ we obtain

$$
I_{10}:=\int_{Q_{\tau}} \sum_{i, j=1}^{n} a_{i j}(x) u_{x_{i}}^{s} v_{x_{j}} d x d t:=I_{10}^{1}+I_{10}^{2},
$$

where

$$
\begin{aligned}
I_{10}^{1}:= & \int_{Q_{\tau}} \sum_{i, j=1}^{n} a_{i j}(x) u_{x_{i}}^{s} u_{t x_{j}}^{s} \Psi_{R}(x) e^{-\eta t} d x d t \geq \frac{a_{1}}{2} \int_{\Omega_{\tau}^{R}} \sum_{i=1}^{n}\left|u_{x_{i}}^{s}\right|^{2} \Psi_{R}(x) e^{-\eta \tau} d x \\
& +\frac{a_{1} \eta}{2} \int_{Q_{\tau}^{R}} \sum_{i=1}^{n}\left|u_{x_{i}}^{s}\right|^{2} \Psi_{R}(x) e^{-\eta t} d x d t-\frac{1}{2} \int_{\Omega_{0}} \sum_{i, j=1}^{n} a_{i j}(x) u_{0, s x_{i}}^{s} u_{0, s x_{j}}^{s} \Psi_{R}(x) d x .
\end{aligned}
$$

By analogy to $I_{9}^{2}$, we have

$$
\begin{aligned}
I_{10}^{2} & :=\int_{Q_{\tau}} \sum_{i, j=1}^{n} a_{i j}(x) u_{x_{i}}^{s} u_{t}^{s} \Psi_{R x_{j}}(x) e^{-\eta t} d x d t \\
& \leq\left(\int _ { Q _ { \tau } } \sum _ { i , j = 1 } ^ { n } \left(\underset{\Omega}{\left.\left.\operatorname{ess} \sup _{\Omega}\left(a_{i j}(x)\right)\right)^{2}\left|u_{x_{i}}^{s}\right|^{2} \Psi_{R}(x) e^{-\eta t} d x d t\right)^{\frac{1}{2}}}\right.\right.
\end{aligned}
$$




$$
\begin{aligned}
& \times\left(\int_{Q_{\tau}}\left|u_{t}^{s}\right|^{q} \Psi_{R}(x) e^{-\eta t} d x d t\right)^{\frac{1}{q}} \cdot\left(\int_{Q_{\tau}} \sum_{j=1}^{n}\left(\frac{\Psi_{R x_{j}}}{\Psi_{R}^{\frac{1}{2}+\frac{1}{q}}}\right)^{r_{1}} e^{-\eta t} d x d t\right)^{\frac{1}{r_{1}}} \\
\leq & \frac{\delta_{2}}{2} \int_{Q_{\tau}} \sum_{i=1}^{n}\left|u_{x_{i}}^{s}\right|^{2} \Psi_{R}(x) e^{-\eta t} d x d t+\frac{\delta_{2}}{2 q} \int_{Q_{\tau}}\left|u_{t}^{s}\right|^{q} \Psi_{R}(x) e^{-\eta t} d x d t \\
& +\mu_{2}\left(\delta_{2}\right) \int_{Q_{\tau}} \sum_{j=1}^{n}\left(\frac{\Psi_{R x_{j}}}{\Psi_{R}^{\frac{1}{2}+\frac{1}{q}}}\right)^{r_{1}} e^{-\eta t} d x d t .
\end{aligned}
$$

Next, from the assumption (B) we have

$$
I_{11}:=\int_{Q_{\tau}} \sum_{i=1}^{n} b_{i}(x)\left|u_{t x_{i}}^{s}\right|^{p-2} u_{t x_{i}}^{s} v_{x_{i}} d x d t:=I_{11}^{1}+I_{11}^{2},
$$

where

$$
\begin{aligned}
I_{11}^{1} & :=\int_{Q_{\tau}} \sum_{i=1}^{n} b_{i}(x)\left|u_{t x_{i}}^{s}\right|^{p-2} u_{t x_{i}}^{s} u_{t x_{i}}^{s} \Psi_{R}(x) e^{-\eta t} d x d t \\
& \geq b_{0} \int_{Q_{\tau}} \sum_{i=1}^{n}\left|u_{t x_{i}}^{s}\right|^{p} \Psi_{R}(x) e^{-\eta t} d x d t .
\end{aligned}
$$

Using Hölder's and Young's inequalities by analogy to $I_{9}^{2}$ we obtain

$$
\begin{aligned}
I_{11}^{2}:= & \int_{Q_{\tau}} \sum_{i=1}^{n} b_{i}(x)\left|u_{t x_{i}}^{s}\right|^{p-2} u_{t x_{i}}^{s} u_{t}^{s} \Psi_{R x_{i}}(x) e^{-\eta t} d x d t \\
\leq & \left(\int_{Q_{\tau}} \sum_{i=1}^{n}\left|b_{i}(x)\right|^{p^{\prime}}\left|u_{t x_{i}}^{s}\right|^{(p-1) p^{\prime}} \Psi_{R}(x) e^{-\eta t} d x d t\right)^{\frac{1}{p^{\prime}}} \\
& \cdot\left(\int_{Q_{\tau}}\left|u_{t}^{s}\right|^{q} \Psi_{R}(x) e^{-\eta t} d x d t\right)^{\frac{1}{q}} \cdot\left(\int_{Q_{\tau}} \sum_{i=1}^{n}\left(\frac{\Psi_{R x_{i}}}{\Psi_{R}^{\frac{1}{p^{\prime}}+\frac{1}{q}}}\right)^{r_{2}} e^{-\eta t} d x d t\right)^{\frac{1}{r_{2}}} \\
\leq & \frac{b_{0}^{1} \delta_{2}}{2 p^{\prime}} \int_{Q_{\tau}} \sum_{i=1}^{n}\left|u_{t x_{i}}^{s}\right|{ }^{(p-1) p^{\prime}} \Psi_{R}(x) e^{-\eta t} d x d t+\frac{\delta_{2}}{2 q} \int_{Q_{\tau}}\left|u_{t}^{s}\right|^{q} \Psi_{R}(x) e^{-\eta t} d x d t \\
& +\mu_{3}\left(\delta_{2}\right) \int_{Q_{\tau}} \sum_{i=1}^{n}\left(\frac{\Psi_{R x_{i}}}{\Psi_{R}^{\frac{1}{p^{\prime}}+\frac{1}{q}}}\right)^{r_{2}} e^{-\eta t} d x d t,
\end{aligned}
$$


where $r_{2}=\frac{p^{\prime} q}{q p^{\prime}-p^{\prime}-q}, b_{0}^{1}=\max _{i=1, \ldots n} \operatorname{ess} \sup _{\Omega}\left|b_{i}(x)\right|^{p^{\prime}}$. From $\left(\mathbf{A}_{\mathbf{3}}\right)$ we obtain

$$
\begin{aligned}
I_{12}:= & \int_{Q_{\tau}} a_{0}(x) u^{s} u_{t}^{s} \Psi_{R}(x) e^{-\eta t} d x d t=\frac{1}{2} \int_{\Omega_{\tau}} a_{0}(x)\left|u^{s}\right|^{2} \Psi_{R}(x) e^{-\eta \tau} d x \\
& -\frac{1}{2} \int_{\Omega_{0}} a_{0}(x)\left|u_{0, s}^{s}\right|^{2} \Psi_{R}(x) d x+\frac{\eta}{2} \int_{Q_{\tau}} a_{0}(x)\left|u^{s}\right|^{2} \Psi_{R}(x) e^{-\eta t} d x d t \\
\geq & -\frac{1}{2} \int_{\Omega_{0}} a_{0}(x)\left|u_{0, s}^{s}\right|^{2} d x+\frac{a_{0}}{2} \int_{\Omega_{\tau}}\left|u^{s}\right|^{2} \Psi_{R}(x) e^{-\eta \tau} d x \\
& +\frac{a_{0} \eta}{2} \int_{Q_{\tau}}\left|u^{s}\right|^{2} \Psi_{R}(x) e^{-\eta t} d x d t .
\end{aligned}
$$

From (C) we have

$$
\begin{aligned}
I_{13} & :=\int_{Q_{\tau}} c(x)\left|u_{t}^{s}\right|^{q-2} u_{t}^{s} u_{t}^{s} \Psi_{R}(x) e^{-\eta t} d x d t \geq c_{0} \int_{Q_{\tau}}\left|u_{t}^{s}\right|^{q} \Psi_{R}(x) e^{-\eta t} d x d t \\
I_{14} & :=\int_{Q_{\tau}} f^{s}(x, t) u_{t}^{s} \Psi_{R}(x) e^{-\eta t} d x d t \\
& \leq \frac{1}{2 \delta_{2}} \int_{Q_{\tau}}\left|f^{s}(x, t)\right|^{2} \Psi_{R}(x) e^{-\eta t} d x d t+\frac{\delta_{2}}{2} \int_{Q_{\tau}}\left|u_{t}^{s}\right|^{2} \Psi_{R}(x) e^{-\eta t} d x d t
\end{aligned}
$$

where $\delta_{2}>0$. Let $R_{0}, 1<R_{0}<R$ be fixed. Thus from (2.16), taking into account the estimates of the integrals $I_{8}-I_{14}$ and assumptions of Theorem 2.4, we get the following inequality

$$
\begin{aligned}
& \int_{\Omega_{\tau}}\left[\left|u_{t}^{s}\right|^{2}+a_{2} \sum_{i, j=1}^{n}\left|u_{x_{i} x_{j}}^{s}\right|^{2}+a_{1} \sum_{i=1}^{n}\left|u_{x_{i}}^{s}\right|^{2}+a_{0}\left|u^{s}\right|^{2}\right] \Psi_{R}(x) e^{-\eta \tau} d x \\
& +\int_{Q_{\tau}}\left[\left(\eta-\delta_{2}\right)\left|u_{t}^{s}\right|^{2}+\left(\eta a_{2}-2 a_{2}^{0} \delta_{2}\right) \sum_{i, j=1}^{n}\left|u_{x_{i} x_{j}}^{s}\right|^{2}+\left(\eta a_{1}-\delta_{2}\right) \sum_{i=1}^{n}\left|u_{x_{i}}^{s}\right|^{2}\right. \\
& +\left(2 b_{0}-\frac{\delta_{2}}{p}-\frac{b_{0}^{1} \delta_{2}}{p^{\prime}}\right) \sum_{i=1}^{n}\left|u_{t x_{i}}^{s}\right|^{p}+\left(2 c_{0}-\frac{3 \delta_{2}}{q}\right)\left|u_{t}^{s}\right|^{q} \\
& \left.+a_{0} \eta\left|u^{s}\right|^{2}\right] \Psi_{R}(x) e^{-\eta t} d x d t \leq \frac{1}{\delta_{2}} \int_{Q_{\tau}}\left|f^{s}(x, t)\right|^{2} \Psi_{R}(x) e^{-\eta t} d x d t \\
& +\int_{\Omega_{0}}\left[\left|u_{1, s}^{s}\right|^{2}+\sum_{i, j, k, l=1}^{n} a_{i j}^{k l}(x) u_{0, s x_{i} x_{j}}^{s} u_{0, s x_{k} x_{l}}^{s}\right.
\end{aligned}
$$




$$
\begin{aligned}
& \left.+\sum_{i, j=1}^{n} a_{i j}(x) u_{0, s x_{i}}^{s} u_{0, s x_{j}}^{s}+a_{0}(x)\left|u_{0, s}^{s}\right|^{2}\right] \Psi_{R}(x) d x \\
& +\mu_{4}\left(\delta_{2}\right) \int_{Q_{\tau}} \sum_{i=1}^{n}\left(\frac{\Psi_{R x_{i}}}{\Psi_{R}^{\frac{1}{p^{\prime}}+\frac{1}{q}}}\right)^{r_{2}} e^{-\eta t} d x d t
\end{aligned}
$$

where $\left(\frac{\Psi_{R x_{i}}}{\Psi_{R}^{\frac{1}{p^{\prime}}+\frac{1}{q}}}\right)^{r_{2}} \leq C \cdot\left(\left[h_{R}(x)\right]^{-r_{2}+\left(1-\frac{1}{p^{\prime}}-\frac{1}{q}\right) r_{2} \beta}\right) \leq C(2 R)^{-r_{2}+\beta}$. From $(2.17)$ and the form of the function $h_{R}$ we have

$$
\Psi_{R}(x)=\left[\frac{1}{R}(R-|x|)(R+|x|)\right]^{\beta} \geq\left(R-R_{0}\right)^{\beta} \quad \text { for }|x| \leq R_{0}
$$

We choose $\delta_{2}, \eta$ from the inequalities $\left(\eta-\delta_{2}\right) \geq 1, \eta a_{2}^{0}-2 \delta_{2} a_{0}^{2} \geq 1,2 b_{0}-\frac{\delta_{2}}{p}-\frac{b_{0}^{1} \delta_{2}}{p^{\prime}} \geq$ $b_{0}, 2 c_{0}-\frac{3 \delta_{2}}{q} \geq c_{0}, \eta a_{1}-\delta_{2} \geq 1$.

Then from (2.18) we get that the sequence $\left\{u^{s}\right\}$ is bounded in $L^{\infty}((0, T)$; $\left.H_{0,1}^{2}\left(\Omega^{R_{0}}\right)\right),\left\{u_{t}^{s}\right\}$ is bounded in $L^{p}\left((0, T) ; W_{0,1}^{p}\left(\Omega^{R_{0}}\right)\right) \cap L^{q}\left(Q_{T}^{R_{0}}\right)$ and from $(2.13)$ it follows that $\left\{u_{t t}^{s}\right\}$ is bounded in $L^{p^{\prime}}\left((0, T) ; W_{0}^{-1, p}\left(\Omega^{R_{0}}\right)\right)+L^{q^{\prime}}\left(Q_{T}^{R_{0}}\right)$. As for as $L^{p^{\prime}}\left((0, T) ; W_{0}^{-1, p}\left(\Omega^{R_{0}}\right)\right)+L^{q^{\prime}}\left(Q_{T}^{R_{0}}\right) \subset L^{q^{\prime}}\left((0, T) ; W_{0}^{-1, p}\left(\Omega^{R_{0}}\right)\right)$ and the imbed$\operatorname{ding} W_{0,1}^{p}\left(\Omega^{R_{0}}\right) \subset L^{p}\left(\Omega^{R}\right)$ is compact then by [20, p.I,s.5.2] the sequence $\left\{u_{t}^{s}\right\}$ is compact in $L^{p}\left(Q_{T}^{R_{0}}\right)$.

Now we can choose the sequence of domain $Q_{T}^{R_{0}}$ such that $Q_{T}^{R_{0}} \subset Q_{T}^{R_{0}+1}$, $R_{0}=2,3, \ldots$. From the last inequality we have that the sequence $\left\{u^{s}\right\}$ is bounded in every bounded domain $Q^{R_{0}}$ in the space $L^{2}\left((0, T) ; H_{0,1}^{2}\left(\Omega^{R_{0}}\right)\right),\left\{u_{t}^{s}\right\}$ is bounded in $L^{p}\left((0, T) ; W_{0,1}^{1, p}\left(\Omega^{R_{0}}\right)\right) \cap L^{q}\left(Q_{T}^{R_{0}}\right),\left\{u_{t t}^{s}\right\}$ is bounded in $L^{q^{\prime}}\left((0, T) ; W_{0}^{-1, p}\left(\Omega^{R_{0}}\right)\right)$. Hence we can construct the diagonal subsequence $\left\{u^{s s}\right\}$ such that

$$
\begin{aligned}
& u^{s, s} \rightarrow u \text { weakly in } L^{2}\left((0, T) ; H_{0, l o c}^{2}(\bar{\Omega})\right), \\
& u_{t}^{s, s} \rightarrow u_{t} \text { weakly in } L^{p}\left((0, T) ; W_{0, l o c}^{1, p}(\bar{\Omega})\right) \cap L^{q}\left((0, T) ; L_{l o c}^{q}(\bar{\Omega})\right), \\
& u_{t}^{s, s} \rightarrow u_{t} \text { in } L^{p}\left((0, T) ; L_{l o c}^{p}(\bar{\Omega})\right)
\end{aligned}
$$

as $s \rightarrow \infty$. Now, we can prove that $\left\{u^{s, s}\right\}$ is a Cauchy sequence.

Let $s, k \in N$ and $s>k>R, R>R_{1}>1$. Let us denote $u^{s k}=u^{s, s}-u^{k, k}$. It is easy to show that for every $\tau \in(0, T]$ the following equality is satisfied 


$$
\begin{aligned}
& \int_{Q_{\tau}}\left[u_{t t}^{s k} v+\sum_{i, j, k, l=1}^{n} a_{i j}^{k l}(x) u_{x_{i} x_{j}}^{s k} v_{x_{k} x_{l}}+\sum_{i, j=1}^{n} a_{i j}(x) u_{x_{i}}^{s k} v_{x_{j}}\right. \\
& \quad+\sum_{i=1}^{n}\left[b_{i}(x)\left|u_{t x_{i}}^{s, s}\right|^{p-2} u_{t x_{i}}^{s, s} v_{x_{i}}-b_{i}(x)\left|u_{t x_{i}}^{k, k}\right|^{p-2} u_{t x_{i}}^{k, k} v_{x_{i}}+a_{0}(x) u^{s k} v\right] \\
& \left.+c(x)\left(\left|u_{t}^{s, s}\right|^{q-2} u_{t}^{s, s}-\left|u_{t}^{k, k}\right|^{q-2} u_{t}^{k, k}\right) v-\left(f^{s}(x, t)-f^{k}(x, t)\right) v\right] d x d t=0,
\end{aligned}
$$

where $v=u_{t}^{s k} \Psi_{R}(x) e^{-\alpha t}$.

If we consider the respective components of the equality (2.19), by analogy to $I_{8}-I_{10}, I_{12}$ we will obtain $I_{15}:=\int_{Q_{\tau}} u_{t t}^{s k} u_{t}^{s k} \Psi_{R}(x) e^{-\alpha t} d x d t=\frac{1}{2} \int_{\Omega_{\tau}}\left|u_{t}^{s k}\right|^{2} \Psi_{R}(x)$ $e^{-\alpha \tau} d x+\frac{\alpha}{2} \int_{Q_{\tau}}\left|u_{t}^{s k}\right|^{2} \Psi_{R}(x) e^{-\alpha t} d x d t$. Next, let $I_{16}:=\int_{Q_{\tau}} \sum_{i, j, k, l=1}^{n} a_{i j}^{k l}(x) u_{x_{i} x_{j}}^{s k} v_{x_{k} x_{l}}$ $d x d t=I_{16}^{1}+I_{16}^{2}+I_{16}^{3}$, where

$$
\begin{aligned}
I_{16}^{1}:= & \int_{Q_{\tau}} \sum_{i, j, k, l=1}^{n} a_{i j}^{k l}(x) u_{x_{i} x_{j}}^{s k} u_{t x_{k} x_{l}}^{s k} \Psi_{R}(x) e^{-\alpha t} d x d t \\
\geq & \frac{a_{2}}{2} \int_{\Omega_{\tau}} \sum_{i, j=1}^{n}\left|u_{x_{i} x_{j}}^{s k}\right|^{2} \Psi_{R}(x) e^{-\alpha \tau} d x \\
& -\frac{1}{2} \int_{\Omega_{0}} \sum_{i, j, k, l=1}^{n} a_{i j}^{k l}(x) u_{0, s x_{i} x_{j}}^{s k} u_{0, s x_{k} x_{l}}^{s k} \Psi_{R}(x) d x \\
& +\frac{\alpha a_{2}}{2} \int_{Q_{\tau}} \sum_{i, j=1}^{n}\left|u_{x_{i} x_{j}}^{s k}\right|^{2} \Psi_{R}(x) e^{-\alpha t} d x d t \\
\geq & \frac{a_{2}}{2} \int_{\Omega_{\tau}} \sum_{i, j=1}^{n}\left|u_{x_{i} x_{j}}^{s k}\right|^{2} \Psi_{R}(x) e^{-\alpha \tau} d x \\
& +\frac{\alpha a_{2}}{2} \int_{Q_{\tau}} \sum_{i, j=1}^{n}\left|u_{x_{i} x_{j}}^{s k}\right|^{2} \Psi_{R}(x) e^{-\alpha t} d x d t, \\
I_{16}^{2} \leq & \frac{\delta}{2} a_{2}^{0} \int_{\Omega_{\tau}} \sum_{i, j=1}^{n}\left|u_{x_{i} x_{j}}^{s k}\right|^{2} \Psi_{R}(x) e^{-\alpha t} d x d t \\
& +\frac{\delta}{2 p} \int_{Q_{\tau}} \sum_{k=1}^{n}\left|u_{x_{k}}^{s k}\right|^{p} \Psi_{R}(x) e^{-\alpha t} d x d t \\
& +\mu(\delta) \int_{Q_{\tau}} \sum_{l=1}^{n}\left(\frac{\Psi_{R x_{l}}}{\Psi_{R}^{\frac{1}{2}+\frac{1}{p}}}\right)^{r} e^{-\alpha t} d x d t,
\end{aligned}
$$




$$
\begin{aligned}
I_{16}^{3}:= & \int_{Q_{\tau}} \sum_{i, j, k, l=1}^{n} a_{i j}^{k l}(x) u_{x_{i} x_{j}}^{s k} u_{t}^{s k} \Psi_{R x_{l}, x_{k}}(x) e^{-\alpha t} d x d t \\
\leq & \frac{a_{2}^{0} \delta}{2} \int_{Q_{\tau}} \sum_{i, j=1}^{n}\left|u_{x_{i} x_{j}}^{s k}\right|^{2} \Psi_{R}(x) e^{-\alpha t} d x d t \\
& +\frac{\delta}{2 q} \int_{Q_{\tau}}\left|u_{t}^{s k}\right|^{q} \Psi_{R}(x) e^{-\alpha t} d x d t \\
& +\mu_{1}(\delta) \int_{Q_{\tau}} \sum_{k, l=1}^{n}\left(\frac{\Psi_{R x_{l} x_{k}}}{\Psi_{R}^{\frac{1}{2}+\frac{1}{q}}}\right)^{r_{1}} e^{-\alpha t} d x d t, \quad \delta>0 .
\end{aligned}
$$

From $I_{10}$ we obtain $I_{17}:=\int_{Q_{\tau}} \sum_{i, j=1}^{n} a_{i j}(x) u_{x_{i}}^{s k} v_{x_{j}} d x d t=I_{17}^{1}+I_{17}^{2}$, where

$$
\begin{aligned}
I_{17}^{1}:= & \int_{Q_{\tau}} \sum_{i, j=1}^{n} a_{i j}(x) u_{x_{i}}^{s k} u_{t x_{j}}^{s k} \Psi_{R}(x) e^{-\alpha t} d x d t \\
\geq & \frac{a_{1}}{2} \int_{\Omega_{\tau}^{R}} \sum_{i=1}^{n}\left|u_{x_{i}}^{s k}\right|^{2} \Psi_{R}(x) e^{-\alpha \tau} d x \\
& +\frac{a_{1} \alpha}{2} \int_{Q_{\tau}^{R}} \sum_{i=1}^{n}\left|u_{x_{i}}^{s k}\right|^{2} \Psi_{R}(x) e^{-\alpha t} d x d t \quad \text { and } \\
I_{17}^{2}:= & \int_{Q_{\tau}} \sum_{i, j=1}^{n} a_{i j}(x) u_{x_{i}}^{s k} u_{t}^{s k} \Psi_{R x_{j}}(x) e^{-\alpha t} d x d t \\
\leq & \frac{\delta}{2} \int_{Q_{\tau}} \sum_{i=1}^{n}\left|u_{x_{i}}^{s k}\right|^{2} \Psi_{R}(x) e^{-\alpha t} d x d t \\
& +\frac{\delta}{2 q} \int_{Q_{\tau}}\left|u_{t}^{s k}\right|^{q} \Psi_{R}(x) e^{-\alpha t} d x d t \\
& +\mu_{2}(\delta) \int_{Q_{\tau}} \sum_{j=1}^{n}\left(\frac{\Psi_{R x_{j}}}{\Psi_{R}^{\frac{1}{2}+\frac{1}{q}}}\right)^{r_{1}} e^{-\alpha t} d x d t .
\end{aligned}
$$

Now, we consider the following component

$$
\begin{aligned}
I_{18}:= & \int_{Q_{\tau}} \sum_{i=1}^{n} b_{i}(x)\left(\left|u_{t x_{i}}^{s, s}\right|^{p-2} u_{t x_{i}}^{s, s}-\left|u_{t x_{i}}^{k, k}\right|^{p-2} u_{t x_{i}}^{k, k}\right) v_{x_{i}} d x d t \\
= & \int_{Q_{\tau}} \sum_{i=1}^{n} b_{i}(x)\left(\left|u_{t x_{i}}^{s, s}\right|^{p-2} u_{t x_{i}}^{s, s}-\left|u_{t x_{i}}^{k, k}\right|^{p-2} u_{t x_{i}}^{k, k}\right) u_{t x_{i}}^{s k} \Psi_{R}(x) e^{-\alpha t} d x d t \\
& +\int_{Q_{\tau}} \sum_{i=1}^{n} b_{i}(x)\left(\left|u_{t x_{i}}^{s, s}\right|^{p-2} u_{t x_{i}}^{s, s}-\left|u_{t x_{i}}^{k, k}\right|^{p-2} u_{t x_{i}}^{k, k}\right) u_{t}^{s k} \Psi_{R x_{i}}(x) e^{-\alpha t} d x d t \\
= & I_{18}^{1}+I_{18}^{2},
\end{aligned}
$$


where

$$
\begin{aligned}
I_{18}^{1}: & =\int_{Q_{\tau}} \sum_{i=1}^{n} b_{i}(x)\left(\left|u_{t x_{i}}^{s, s}\right|^{p-2} u_{t x_{i}}^{s, s}-\left|u_{t x_{i}}^{k, k}\right|^{p-2} u_{t x_{i}}^{k, k}\right) \cdot u_{t x_{i}}^{s k} \Psi(x) e^{-\alpha t} d x d t \\
\geq & 2^{2-p} b_{0} \int_{Q_{\tau}} \sum_{i=1}^{n}\left|u_{t x_{i}}^{s k}\right|^{p} \Psi_{R}(x) e^{-\alpha t} d x d t \text { and } \\
I_{18}^{2}:= & \int_{Q_{\tau}} \sum_{i=1}^{n} b_{i}(x)\left(\left|u_{t x_{i}}^{s, s}\right|^{p-2} u_{t x_{i}}^{s, s}-\left|u_{t x_{i}}^{k, k}\right|^{p-2} u_{t x_{i}}^{k, k}\right) u_{t}^{s k} \Psi_{R x_{i}}(x) e^{-\alpha t} d x d t \\
\leq & \left(\left.\int_{Q_{\tau}} \sum_{i=1}^{n}\left|b_{i}(x)\right|^{p^{\prime}}|| u_{t x_{i}}^{s, s}\right|^{p-2} u_{t x_{i}}^{s, s}-\left.\left|u_{t x_{i}}^{k, k}\right|^{p-2} u_{t x_{i}}^{k, k}\right|^{p^{\prime}}\left|\Psi_{R x_{i}}(x)\right| e^{-\alpha t} d x d t\right)^{\frac{1}{p^{\prime}}} \\
& \times\left(\int_{Q_{\tau}} \sum_{i=1}^{n}\left|u_{t}^{s k}\right|^{p}\left|\Psi_{R x_{i}}(x)\right| e^{-\alpha t} d x d t\right)^{\frac{1}{p}} \equiv p_{s k} .
\end{aligned}
$$

We can choose $k_{0}>R+1$ such that for all $s, k \geq k_{0}, p_{s k}$ will be small enough. From $I_{12}$ we obtain

$$
\begin{aligned}
I_{19}:= & \int_{Q_{\tau}} a_{0}(x) u^{s k} u_{t}^{s k} \Psi_{R}(x) e^{-\alpha t} d x d t \geq-\frac{1}{2} \int_{\Omega_{0}} a_{0}(x)\left|u_{0, s}^{s k}\right|^{2} \Psi_{R}(x) d x \\
& +\frac{a_{0}}{2} \int_{\Omega_{\tau}}\left|u^{s k}\right|^{2} \Psi_{R}(x) e^{-\alpha \tau} d x+\frac{a_{0} \alpha}{2} \int_{Q_{\tau}}\left|u^{s k}\right|^{2} \Psi_{R}(x) e^{-\alpha t} d x d t \\
\geq & \frac{a_{0}}{2} \int_{\Omega_{\tau}}\left|u^{s k}\right|^{2} \Psi_{R}(x) e^{-\alpha \tau} d x+\frac{a_{0} \alpha}{2} \int_{Q_{\tau}}\left|u^{s k}\right|^{2} \Psi_{R}(x) e^{-\alpha t} d x d t .
\end{aligned}
$$

Similarly to $I_{18}^{1}$ from $(\mathbf{C})$ we have

$$
\begin{aligned}
I_{20} & :=\int_{Q_{\tau}} c(x)\left(\left|u_{t}^{s, s}\right|^{q-2} u_{t}^{s, s}-\left|u_{t}^{k, k}\right|^{q-2} u_{t}^{k, k}\right) \cdot u_{t}^{s k} \Psi_{R}(x) e^{-\alpha t} d x d t \\
& \geq c_{0} 2^{2-q} \int_{Q_{\tau}}\left|u_{t}^{s k}\right|^{q} \Psi_{R}(x) e^{-\alpha t} d x d t .
\end{aligned}
$$

From estimates of the integrals $I_{15}-I_{20}$ and from the initial condition the following inequality is satisfied 


$$
\begin{aligned}
& \int_{\Omega_{\tau}^{R_{1}}}\left[\left|u_{t}^{s k}\right|^{2}+a_{2} \sum_{i, j=1}^{n}\left|u_{x_{i} x_{j}}^{s k}\right|^{2}+a_{1} \sum_{i=1}^{n}\left|u_{x_{i}}^{s k}\right|^{2}+a_{0}\left|u^{s k}\right|^{2}\right] e^{-\alpha \tau} d x \\
& +\int_{Q_{\tau}^{R_{1}}}\left[\alpha\left|u_{t}^{s k}\right|^{2}+\left(\alpha a_{2}-2 a_{2}^{0} \delta\right) \sum_{i, j=1}^{n}\left|u_{x_{i} x_{j}}^{s k}\right|^{2}+\left(\alpha a_{1}-\delta\right) \sum_{i=1}^{n}\left|u_{x_{i}}^{s k}\right|^{2}\right. \\
& +\left(2^{3-p^{2}} b_{0}-\frac{\delta}{p}\right) \sum_{i=1}^{n}\left|u_{t x_{i}}^{s k}\right|^{p}+\left(2^{3-q} c_{0}-\frac{\delta}{q}\right)\left|u_{t}^{s k}\right|^{q} \\
& \left.+a_{0} \alpha\left|u^{s k}\right|^{2}\right] e^{-\alpha t} d x d t \leq \frac{2}{\left(R-R_{1}\right)^{\beta}}\left\{\int _ { Q _ { \tau } } \left[\mu(\delta) \sum_{l=1}^{n}\left(\frac{\Psi_{R x_{l}}}{\Psi_{R}^{\frac{1}{2}+\frac{1}{p}}}\right)^{r}\right.\right. \\
& \left.\left.+\mu_{1}(\delta) \sum_{k, l=1}^{n}\left(\frac{\Psi_{R x_{l} x_{k}}}{\Psi_{R}^{\frac{1}{2}+\frac{1}{q}}}\right)^{r_{1}}+\mu_{2}(\delta) \sum_{j=1}^{n}\left(\frac{\Psi_{R x_{j}}}{\Psi_{R}^{\frac{1}{2}+\frac{1}{q}}}\right)^{r_{1}}\right] e^{-\alpha t} d x d t+p_{s k}\right\}
\end{aligned}
$$

We can choose $\alpha$ and $\delta$ such that the inequalities $\alpha a_{2}-2 a_{2}^{0} \delta \geq 1,2^{3-p} b_{0}-\frac{\delta}{p} \geq$ $2^{2-p} b_{0}, 2^{3-q} c_{0}-\frac{\delta}{q} \geq 2^{2-q} c_{0}, \alpha a_{1}-\delta \geq 1$ hold. Moreover form (2.20) we have

$$
\left(\frac{\Psi_{R x_{i}}}{\Psi_{R}^{\frac{1}{2}+\frac{1}{p}}}\right)^{r} \leq C(2 R)^{-r+\beta}, \quad\left(\frac{\Psi_{R x_{i}}}{\Psi_{R}^{\frac{1}{2}+\frac{1}{q}}}\right)^{r_{1}} \leq C(2 R)^{-r_{1}+\beta} .
$$

Next, since $\left|\Psi_{R x_{l} x_{k}}\right| \leq C \cdot \Psi_{R}\left[h_{R}\right]^{-2}$, we get $\left(\frac{\Psi_{R x_{k} x_{l}}}{\Psi_{R}^{\frac{1}{2}+\frac{1}{q}}}\right)^{r_{1}} \leq C(2 R)^{-2 r_{1}+\beta}$. Hence

$$
\begin{aligned}
& \mu(\delta) \int_{Q_{\tau}} \sum_{l=1}^{n}\left(\frac{\Psi_{R x_{l}}}{\Psi_{R}^{\frac{1}{2}+\frac{1}{p}}}\right)^{r} e^{-\alpha t} d x d t+\mu_{1}(\delta) \int_{Q_{\tau}} \sum_{k, l=1}^{n}\left(\frac{\Psi_{R x_{l} x_{k}}}{\Psi_{R}^{\frac{1}{2}+\frac{1}{q}}}\right)^{r_{1}} e^{-\alpha t} d x d t \\
& \quad+\mu_{2}(\delta) \int_{Q_{\tau}} \sum_{j=1}^{n}\left(\frac{\Psi_{R x_{j}}}{\Psi_{R}^{\frac{1}{2}+\frac{1}{q}}}\right)^{r_{1}} e^{-\alpha t} d x d t \\
& \leq \mu_{1}(\delta) C_{1}(2 R)^{-r+\beta+n}+\mu_{1}(\delta) C_{1}(2 R)^{-2 r_{1}+\beta+n}+\mu_{2}(\delta) C_{1}(2 R)^{-r_{1}+\beta+n} .
\end{aligned}
$$

Let $\epsilon>0$ be an arbitrary small number. From (2.20) we get that there exist $R>0$ and $\kappa_{0}(R)$ such that the right hand of (2.20) is less than $\epsilon$. Hence from (2.20) we have

$$
\left\|u_{t}^{s, s}-u_{t}^{k, k}\right\|_{C\left([0, T] ; L^{2}\left(\Omega^{R}\right)\right)}<\epsilon, \quad\left\|u^{s, s}-u^{k, k}\right\|_{L^{2}\left((0, T) ; H_{0,1}^{2}\left(\Omega^{R}\right)\right)}<\epsilon
$$

and

$$
\left\|u_{t}^{s, s}-u_{t}^{k, k}\right\|_{L^{q}\left(Q_{T}^{R}\right)}<C_{2} \epsilon, \quad\left\|u_{t}^{s, s}-u_{t}^{k, k}\right\|_{L^{p}\left((0, T) ; W_{0,1}^{1, p}\left(\Omega^{R}\right)\right)}<\epsilon .
$$


Thus the diagonal sequence $\left\{u^{s, s}\right\}$ satisfies the Cauchy condition. Taking into account that $R$ is arbitrary, it follows that

$$
\begin{aligned}
& u^{s, s} \rightarrow u \text { in } L^{2}\left((0, T) ; H_{0, \mathrm{loc}}^{2}(\bar{\Omega})\right), \quad u_{t}^{s, s} \rightarrow u_{t} \text { in } C\left([0, T] ; L_{\mathrm{loc}}^{2}(\bar{\Omega})\right), \\
& u^{s, s} \rightarrow u \text { in } L^{p}\left((0, T) ; W_{0, \mathrm{loc}}^{1, p}(\bar{\Omega})\right) \cap L^{q}\left((0, T) ; L_{\mathrm{loc}}^{q}(\bar{\Omega})\right) .
\end{aligned}
$$

If we put with $s$ to infinity then from (2.16) we obtain

$$
\begin{aligned}
& \int_{\Omega_{\tau}} u_{t}(x, \tau) v(x, \tau) d x+\int_{Q_{\tau}}\left[-u_{t} v_{t}+\sum_{i, j, k, l=1}^{n} a_{i j}^{k l}(x) u_{x_{i} x_{j}} v_{x_{k} x_{l}}\right. \\
& \quad+\sum_{i, j=1}^{n} a_{i j}(x) u_{x_{i}} v_{x_{j}}+\sum_{i=1}^{n} b_{i}(x)\left|u_{t x_{i}}\right|^{p-2} u_{t x_{i}} v_{x_{i}} \\
& \left.\quad+a_{0}(x) u v+c(x)\left|u_{t}\right|^{q-2} u_{t} v\right] d x d t \\
& =\int_{Q_{\tau}} f(x, t) v d x d t+\int_{\Omega_{0}} u_{1}(x) v(x, 0) d x
\end{aligned}
$$

$\forall v \in L^{2}\left((0, T) ; H_{0, \text { loc }}^{2}(\bar{\Omega})\right) \cap L^{q}\left((0, T) ; L_{\text {loc }}^{q}(\bar{\Omega})\right) \cap L^{p}\left((0, T) ; W_{0, \text { loc }}^{1, p}(\bar{\Omega})\right), v_{t} \in L^{2}$ $\left((0, T) ; L_{\text {loc }}^{2}(\bar{\Omega})\right)$, where supp $v$ is bounded.

Besides that $u^{s, s}(x, 0)=u_{0 s}(x), u_{0 s} \rightarrow u_{0}$ in $L_{\text {loc }}^{2}(\bar{\Omega})$, hence $u(x, 0)=u_{0}(x)$. In virtue of Definition 1 , the function $u$ is a solution of the problem (1.1)-(1.3).

\section{References}

[1] Benilan, Ph., Grandall, M.G., Pierre, M.: Solutions of the porous medium equations in $R^{n}$ under optimal conditions on initial values. Indiana Univ. Math. J. 1, 51-87 (1984)

[2] Bernis, F.: Elliptic and parabolic semilinear parabolic problems without conditions at infinity. Arch. Rational Mech. Anal. 3, 217-241 (1989)

[3] Bokalo, H.M.: Unique solvability of boundary value problems for semilinear parabolic equations in unbounded domains without conditions at infinity. Siberian Math. J. 4, 620-627 (1993)

[4] Bokalo, H.M.: Boundary value problems for semilinear parabolic equations in unbounded domains without conditions at infinity. Siberian Math. J. 5, 860867 (1996)

[5] Brezis, H.: Semilinear equations in $R^{N}$ without conditions at infinity. Appl. Math. Optim. 3, 271-282 (1984) 
[6] Dahlberg, B.E.J., Kenig, C.E.: Non-negative solutions of the porous medium equation. Commun. Partial Differ. Equ. 5, 409-437 (1984)

[7] Dal Passo, R., Giacomelli, L., Shishov, A.: The thin film equation with nonlinear diffusion. Commun. Partial Differ. Equ. 9-10, 1509-1557 (2001)

[8] Denisow, V.N.: Stabilization of the solution of the Cauchy problem for the iterated Heat equation. Differ. Urawnienia 1, 29-42 (1991)

[9] Denisow, V.N.: Some properties of the solutions at $(t \rightarrow+\infty)$ of the iterated Heat equation. Differ. Urawnienia 1, 59-69 (1992)

[10] Di Benedetto, E., Herrero, M.A.: On the Cauchy problem and initial traces for a degenerate parabolic equation. Trans. Am. Math. Soc. 1, 187-224 (1989)

[11] Di Benedetto, E., Herrero, M.A.: Non-negative solutions of the evolution p-Laplacian equation. Initial traces and Cauchy problem when $1<p<2$. Arch. Rational Mech. Anal. 3, 225-290 (1990)

[12] Gladkov, A.L.: Cauchy problem for equations of heat conductibity with convection in classes of growing functions. Pitman Res. Notes Math. Longman Sci. Tech. Harlow. 325, 106-119 (1995)

[13] Gladkov, A.L.: On the filtration-absorption equation with a variable coefficient. Differ. Urawnienia 1, 45-50 (2001)

[14] Gladkov, A., Guedda, M.: Diffusion-absorption equation without growth restrictions on the data at infinity. J. Math. Anal. Appl. 1, 16-37 (2002)

[15] Gladkov, A., Guedda, M., Prohozhii, S.A.: Cauchy problem for higher-order parabolic equations with arbitrary growing at infinity initial data. Nonlinear Anal. Appl. 1, 563-576 (2001)

[16] Herrero, M.A., Pierre, M.: The Cauchy problem for $u_{t}-\triangle u^{m}=0$ when $0<m<$ 1. Trans. Am. Math. Soc. 1, 145-158 (1985)

[17] Itano, M.: Some remarks on the Cauchy problem for $p$-Parabolic equations. Hiroshima Math. J. 39, 211-228 (1974)

[18] Lavrenyuk, S.P., Protsakh, N.P.: A mixed problem for a nonlinear parabolic equation. Mat. Metodi Fiz.-Mekh. Polya 43(3), 56-63 (2000)

[19] Leonardi, S., Nicolosi, F., Shishkov, A.E.: Cauchy-Dirichlet problem for quasilinear degenerate parabolic equations of higher order with initial data increasing at infinity. Nonlinear World 4, 539-571 (1997)

[20] Lions, J.L.: Quelques méthodes de résolution des probl ems aux limites non linéaires. Dunod, Paris (1969)

[21] Małecki, R., Zielińska, E.: Contour-Integral method applied for solving a certain mixed problem for a parabolic equation of order four. Demonstratio Math. XV.3, 695-709 (1982) 
[22] Marchi, C., Tesei, A.: Higher-order parabolic equations without conditions at infinity. J. Math. Anal. Appl. 1, 352-368 (2002)

[23] McLeod, B., Peletier, L.A., Vazquez, J.L.: Solution of nonlinear ODE appearing in the theory of diffusion with absorption. Differ. Integral Equ. 1, 1-14 (1991)

[24] Mikami, M.: The Cauchy problem for degenerate parabolic equations and Newton Polygon. Funkc. Ekvac. 39, 449-468 (1996)

[25] Oleinik, O.A., Radkievich, E.V.: The method of introducing a parameter for the investigation of evolution equations. Uspekhi Mat. Nauk. 5, 7-76 (1978)

[26] Pierre, M.: Nonlinear fast diffusion with measures as data. Nonlinear parabolic equations: qualitative properties of solutions. Proc. Conf., Rome/Italy 1985. Pitman Research Notes in Mathematics Series, vol. 149, pp. 179-188 (1987)

[27] Protsakh, N.P.: A mixed problem for a nonlinear evolution equation with a second-order time derivative in generalized Lebesque spaces. Mat. Stud. 16(2), 157$168(2001)$

[28] Vazquez, J.L., Walias, M.: Existence and uniqueness of solution of diffusionabsorption equations with general data. Differ. Integral Equ. 1, 15-36 (1994)

L. Zaręba

Institute of Mathematics,

University of Rzeszów,

Rejtana16A,

35-959 Rzeszow, Poland

e-mail: lzareba@univ.rzeszow.pl

Received: 06 June 2008.

Accepted: 25 February 2009. 